\title{
Multi-step evolution and measurement control of finite-dimensional quantum systems
}

\author{
LIU WenFeng, ZHANG ChenBin \& CHEN ZongHai* \\ Department of Automation, University of Science and Technology of China, Hefei 230027, China
}

Received August 23, 2011; accepted December 14, 2011; published online April 24, 2012

\begin{abstract}
For finite-dimensional quantum systems, we propose a quantum control scheme based on a multi-step unitary evolution and quantum projective measurements. The objective is to design a control law to steer the system to a target eigenstate of the measurement operator in the least number of steps. Within each control step, unitary evolution and quantum projective measurement are performed in turn until the system reaches the target state. The control process can be modeled as a finite-state Markov chain with an absorbing state. We prove that the controlled system will converge to the target eigenstate with probability one after a finite number of control steps and find a minimal-step-number condition that would steer the system to the target eigenstate in the least number of steps.
\end{abstract}

quantum control, quantum projective measurement, unitary evolution, Markov chain

Citation: $\quad$ Liu W F, Zhang C B, Chen Z H. Multi-step evolution and measurement control of finite-dimensional quantum systems. Chin Sci Bull, 2012, 57: 2233-2241, doi: 10.1007/s11434-012-5166-3

Since the 1980s, manipulation of quantum systems has become a potentially-profitable but challenging goal [1,2]. A systematic theory on controlling quantum systems is prerequisite in applications such as chemical reactions and quantum information. After almost 30 years of development, researchers have made great strides in many aspects, such as controllability [3], optimal control [4,5], closed-loop learning control [6,7], and quantum feedback control [8]. However, the situation in quantum systems control is very different from controlling classical dynamic systems. For example, quantum systems are difficult to observe and generally are coupled to non-controllable environments. Quantum states are easily destroyed by measurement and decoherence. Quantum measurement appears as one essential obstacle in quantum control because the system state is changed; it is difficult to get feedback information without disturbing the system state.

Since quantum measurement unavoidably disturbs the state of the quantum system, some researchers have pro-

*Corresponding author (email: chenzh@ustc.edu.cn) posed several schemes that employ quantum measurement as an external means to control quantum systems [9-15]. Mendes and Man'ko [9] discussed quantum control in a geometrical setting provided by the Strocchi map. In their paper, quantum time evolution corresponds to smooth Hamiltonian dynamics in a classical-like phase space and measurements correspond to jumps in the phase space. They found that a unitary non-controllable system might become controllable by "measurement plus evolution". Inspired by this idea, Mandilara and Clark [10] proposed a strategy for indirect control of a target system through projective measurements on its entangled partner. Zhang et al. [11] proposed a control scheme based on a single-step quantum measurement and unitary evolution to steer a non-controllable system from one state to another with a probability close to one. Roa et al. [12] studied the problem of mapping an unknown mixed quantum state onto a known pure state by sequential measurements of two non-commuting observables. Pechen et al. [13] presented a scheme for controlling quantum systems by measuring a set of observables one by one until the target was achieved. 
In quantum computing, the state preparation and readout involves transferring a population of discrete quantum states. To transfer this population effectively, several methods have been proposed [16-21]. However, a systematic theory of population transfer is still required. In this paper, our goal for quantum control is to steer the controlled system to an eigenstate of a projective measurement operator. In this case we develop a simple and easy control scheme which only needs to take the same projective measurement after a unitary evolution several times over until the target state is reached. We analyze the control process using the concept Markov chains where the target eigenstate is an absorbing state; we obtain a condition by which the target eigenstate can be reached with the least number of control steps.

The paper is organized as follows. In Section 1, we recall briefly the quantum concepts of evolution and projective measurements. In Section 2, we present our scheme that involves performing quantum unitary evolution and quantum projective measurement in turn until the target eigenstate is achieved. In Section 3, we investigate the scheme where we prove its finite-step reachability with probability one using Markov chains and provide a condition that determines how to reach a target eigenstate with the least number of control steps. Since an analytical solution is very hard to achieve, we analyze in Section 4 the realization of our scheme in various simulations. In Section 5, we give numerical simulations of our scheme for a 2-qubit quantum system. Finally we conclude our results and discuss some potential applications of our scheme in Section 6.

\section{Prerequisites}

\subsection{State representation and evolution}

Quantum mechanics is usually represented in the form of Dirac and matrix notation, where the state of a quantum system is represented by ket, $|\psi\rangle \in \mathcal{H}$, where $\mathcal{H}$ denotes the Hilbert space spanned by all the states of the system. The evolution of the state can be described by the following Schrödinger equation [22]:

$$
i \hbar|\dot{\psi}(t)\rangle=H(\vec{u}(t))|\psi(t)\rangle .
$$

Here, $H(\vec{u}(t))$ represents the Hamiltonian of the system, a Hermitian operator acting on the Hilbert space $\mathcal{H}$ with $\vec{u}(t)$ as a set of external control variables. For a $d$-dimensional quantum system, its state can be expressed as $|\psi\rangle=\sum_{i=1}^{d} \alpha_{i}\left|\psi_{i}\right\rangle$, where $\left\{\left|\psi_{i}\right\rangle\right\}$ forms an orthonormal basis of $\mathcal{H}$. The solution of eq. (1) has the form

$$
|\psi(t)\rangle=U(t)|\psi(0)\rangle,
$$

where $U(t)$ is a unitary evolution operator which can be solved using the Schrödinger operator equation [22]:

$$
i \hbar \dot{U}(t)=H(\vec{u}(t)) U(t), U(0)=I,
$$

where $I$ denotes the identity operator. Eqs. (2) and (3) describe the unitary evolution of a closed finite-dimensional quantum system under external controls $\vec{u}(t)$.

\subsection{Quantum projective measurement}

According to the von Neumann postulates [22,23], when an observable $\mathcal{A}$ is measured, the expectation value corresponds to one of the eigenvalues of $\mathcal{A}$. For a $d$-dimensional quantum system, let $\lambda_{j}(j=1,2, \ldots d)$ denote the eigenvalues of $\mathcal{A}$ with corresponding eigenvectors $\left|\lambda_{j}\right\rangle$. The observable operator can be expressed as

$$
\mathcal{A}=\sum_{j=1}^{d} \lambda_{j}\left|\lambda_{j}\right\rangle\left\langle\lambda_{j}\right|
$$

where $\left|\lambda_{j}\right\rangle\left\langle\lambda_{j}\right|$ can be called as projector $P_{j}$.

Taking the eigenvector set $\left\{\left|\lambda_{j}\right\rangle\right\}$ of the measurement operator as the basis of the state space, then the Hamiltonian and unitary evolution operator can be expressed in matrix form, and any state in the state space can be expressed as a superposition of these eigenstates. If the system before measurement is in state $|\psi\rangle=\sum_{j=1}^{d} \alpha_{j}\left|\lambda_{j}\right\rangle$, then after a projective measurement, the system will collapse to one of the eigenstates $\left|\lambda_{j}\right\rangle$ with probability

$$
\left\langle\psi\left|P_{j}\right| \psi\right\rangle=\left|\alpha_{j}\right|^{2} .
$$

A simple example of a quantum projective measurement is the Stern-Gerlach measurement with neutral spin-1/2 atoms, the spin resulting from an unpaired electron. As depicted in Figure 1, the beam is shot from the source and as it traverses the inhomogeneous magnetic field, the beam splits in two. The observable can be diagonalized as

$$
A=\frac{1}{2}|\uparrow\rangle\left\langle\uparrow\left|-\frac{1}{2}\right| \downarrow\right\rangle\langle\downarrow|
$$

where $|\uparrow\rangle$ and $|\downarrow\rangle$ are eigenstates for spin-up and spin-down respectively. If an atom before measurement is in the superposition state $\left|\psi\left(t_{0}\right)\right\rangle=\alpha_{1}|\uparrow\rangle+\alpha_{2}|\downarrow\rangle$, where $\alpha_{1}$ and $\alpha_{2}$ are complex numbers satisfying $\left|\alpha_{1}\right|^{2}+\left|\alpha_{2}\right|^{2}=1$, after measurement, the atom will be detected by the upper detector with probability $\left|\alpha_{1}\right|^{2}$ and by the lower detector with probability $\left|\alpha_{2}\right|^{2}$.

\section{Multi-step evolution and measurement control}

By combining unitary evolution and quantum projective 


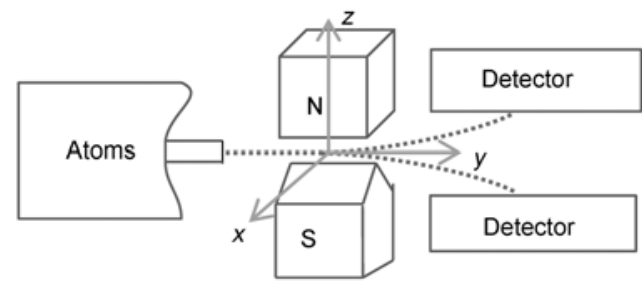

Figure 1 Stern-Gerlach experiment setup for neutral spin-1/2 atoms.

measurements, we propose a method to exercise control over a quantum system and to steer it towards a target eigenstate $\left|\psi_{T}\right\rangle$. Our quantum control scheme works as follows: initially, the system is in an initial state $\left|\psi_{0}\right\rangle=\sum_{j} \alpha_{j}^{0}\left|\lambda_{j}\right\rangle$. We perform a measurement $\mathcal{A}$ on the system. According to the quantum measurement postulate, the system will be in one of the eigenstates $\left|\lambda_{i}\right\rangle$ of $\mathcal{A}$ with a certain probability $\left|\alpha_{i}^{0}\right|^{2}$; if that eigenstate is not equal to $\left|\psi_{T}\right\rangle$, we choose an appropriate evolution time $T^{\lambda_{i}}$ and control variables $\vec{u}^{\lambda_{i}}(t)$ to produce a unitary evolution operator $U^{\lambda_{i}}$ conditioned on the output state $\left|\lambda_{i}\right\rangle$ of the measurement. We subsequently let the system evolve under control variables $\vec{u}^{\lambda_{i}}(t)$ for a period of time $T^{\lambda_{i}}$ with its final state in $|\psi(1)\rangle=U^{\lambda_{i}}\left|\lambda_{i}\right\rangle=\sum_{j} \alpha_{j}^{1}\left|\lambda_{j}\right\rangle$. We perform $\mathcal{A}$ on the system again, and if the output state $\left|\psi^{*}(1)\right\rangle$ is equal to $\left|\psi_{T}\right\rangle$, we have achieved our goal; otherwise, if $\left|\psi^{*}(1)\right\rangle=\left|\lambda_{l}\right\rangle$, we choose an appropriate $U^{\lambda_{l}}$ to evolve the system under control variables $\vec{u}^{\lambda_{l}}(t)$ for a period of time $T^{\lambda_{l}}$, then perform the projective measurement $\mathcal{A}$ once again. We combine each $U^{\lambda_{i}}$ and $\mathcal{A}$ as one control step. If the output state is not equal to the target eigenstate, we repeat the control step until the target eigenstate $\left|\psi_{T}\right\rangle$ is attained.

In each control step, based on the $i$-th output state $\left|\lambda_{i}\right\rangle$ of the last measurement, we choose a unitary evolution operator $U^{\lambda_{i}}$ to apply to the system and then perform the projective measurement that instantly collapses the state to another eigenstate $\left|\lambda_{j}\right\rangle$ with a certain probability. If we focus on just the output state of each measurement, the control process could be described by discrete-time equations:

$$
\begin{gathered}
|\psi(k+1)\rangle=U^{\lambda_{i}}\left|\lambda_{i}\right\rangle \quad \text { for }\left|\psi^{*}(k)\right\rangle=\left|\lambda_{i}\right\rangle, \\
\operatorname{Prob}\left(\left|\psi^{*}(k+1)\right\rangle=\left|\lambda_{j}\right\rangle\right)=\left|\left\langle\lambda_{j} \mid \psi(k+1)\right\rangle\right|^{2},
\end{gathered}
$$

where $|\psi(k)\rangle$ and $\left|\psi^{*}(k)\right\rangle$ are the respective states before and after the projective measurement at step $k$, and $U^{\lambda_{i}}$ is the unitary evolution matrix which is chosen based on the $i$-th output eigenstate from the projective measure- ment.

The control algorithm of the multi-step evolution and measurement control can be described as follows.

(1) Given an initial state $\left|\psi_{0}\right\rangle$ and a target eigenstate $\left|\psi_{T}\right\rangle$.

(2) Apply the projective measurement to the controlled system.

(3) Decide if the output state of the measurement is the target eigenstate $\left|\psi_{T}\right\rangle$, in which event the control process ends; otherwise, go to step (4).

(4) Choose appropriate control variables $\vec{u}^{\lambda_{i}}(t)$ and control period $T^{\lambda_{i}}$ to produce a unitary evolution matrix $U^{\lambda_{i}}$ depending on the output eigenstate $\left|\lambda_{i}\right\rangle$ of the projective measurement.

(5) Let the controlled system evolve under control variables $\vec{u}^{\lambda_{i}}(t)$ for a period of time $T^{\lambda_{i}}$ which corresponds to the unitary evolution matrix $U^{\lambda_{i}}$ chosen in the step (4), then go to step (2).

The whole control process is illustrated in Figure 2 for a 4-dimensional quantum system. Here, the objective is to steer the system from the initial state $\left|\psi_{0}\right\rangle$ to the expected target state $\left|\psi_{T}\right\rangle=\left|\lambda_{4}\right\rangle$. Initially, we perform a projective measurement on the system, collapsing the system to one of its eigenstates $\left|\lambda_{i}\right\rangle$. If $i \neq 4$, let the system evolve under $U^{\lambda_{i}}$ then follow up with the projective measurement. The output state will be in $\left|\lambda_{j}\right\rangle$ with probability $p_{i j}$. If $j=4$, we have achieved the control goal; otherwise, we apply $U^{\lambda_{j}}$ allowing the system to evolve and again take the projective measurement until the target eigenstate $\left|\lambda_{4}\right\rangle$ is achieved.

\section{Markov chain approach}

As inferred from Section 2, our scheme is probabilistic in nature. Hence, there might be situations where, after many (even infinite) control steps, we have not attained the target goal. We shall further investigate the properties of the control process using Markov chains and provide a condition that determines whether we can attain a target state in finite number of steps by the proposed approach.

Proposition 3.1. The multi-step evolution and measurement control scheme is a Markov chain with the target eigenstate as an absorbing state.

Proof. As the initial state is not always one of the eigenstates for the projective operator, we need first to make a projective measurement to return an output eigenstate which we denote by $\left|\psi^{*}(0)\right\rangle$. If it is not the target eigenstate, we perform a controlled evolution followed by the projective measurement. The output eigenstate is denoted as $\left|\psi^{*}(1)\right\rangle$. Analogously, we could express the output eigenstate of step $k$ in our control scheme as $\left|\psi^{*}(k)\right\rangle$ giving with probability $P\left(\left|\psi^{*}(k)\right\rangle=\left|\lambda_{i}\right\rangle\right)=\left|\left\langle\lambda_{i} \mid \psi(k)\right\rangle\right|^{2}$. In this sense, our aim is to 


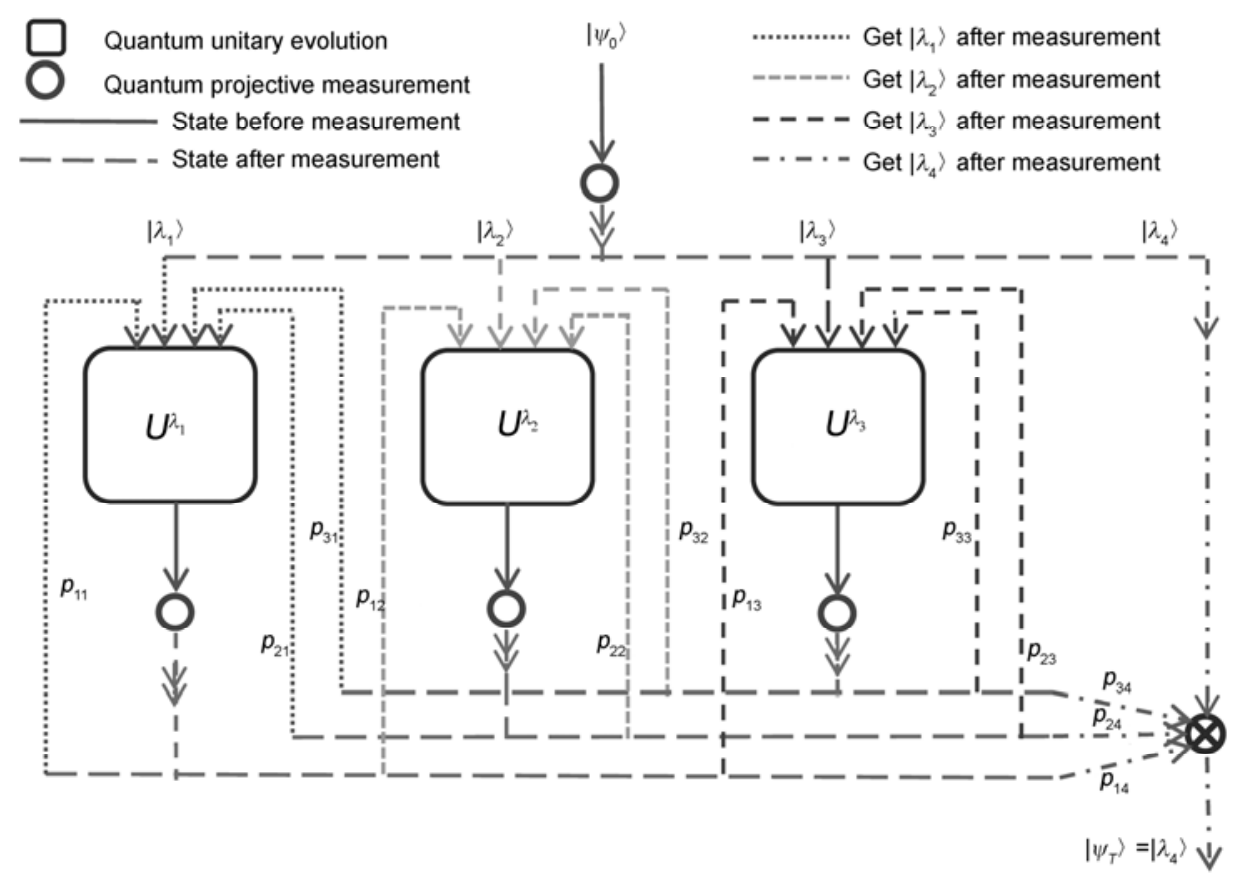

Figure 2 Process of multi-step evolution and measurement control in a 4-dimensional quantum system where the target state is $\left|\lambda_{4}\right\rangle \cdot p_{i j}$ is the transition probability from state $\left|\lambda_{i}\right\rangle$ to state $\left|\lambda_{j}\right\rangle$ in one iteration of a control step. $U^{\lambda i}\{i=1,2,3\}$ is the unitary evolution matrix conditioning on the output eigenstate $\left|\lambda_{i}\right\rangle$ of the projective measurement.

prove that $\left\{\left|\psi^{*}(0)\right\rangle,\left|\psi^{*}(1)\right\rangle,\left|\psi^{*}(2)\right\rangle, \cdots\right\}$ is a Markov chain.

The proof of the above assertion is as follows. The probability of $\left|\psi^{*}(k)\right\rangle=\left|\lambda_{j}\right\rangle \quad$ is $\quad\left|\left\langle\lambda_{j} \mid \psi(k)\right\rangle\right|^{2}=$ $\left|\left\langle\lambda_{j}\left|U^{\psi^{*}(k-1)}\right| \psi^{*}(k-1)\right\rangle\right|^{2}$, where $U^{\psi^{*}(k-1)}$ is the controlled-evolution operator depending on the output eigenstate $\left|\psi^{*}(k-1)\right\rangle$ of the projective measurement. Thus, the probability for collapse to $\left|\psi^{*}(k)\right\rangle=\left|\lambda_{j}\right\rangle$ is only dependent on the state at step $k-1$ and does not depend upon prior states before this step. Hence, the controlled evolution and measurement scheme is a Markov chain.

In this scheme, the target state is one of the eigenstates of the projective operator; if the output state of the measurement is just the target eigenstate, we exit the procedure successfully. From the Markov chain point of view though, the system remains in the target eigenstate and cannot jump to other states. In this sense, the target eigenstate is an absorbing state of the Markov chain $\left\{\left|\psi^{*}(0)\right\rangle\right.$, $\left.\left|\psi^{*}(1)\right\rangle,\left|\psi^{*}(2)\right\rangle, \cdots\right\}$.

Without loss of generality, we assume that the initial state is $\left|\lambda_{1}\right\rangle$ and the target eigenstate is $\left|\lambda_{d}\right\rangle$; if not, we simply reorder the eigenstates. In addition, we assume all operators are represented in the eigenbasis. The state of the Markov chain lies in the space set $\left\{\left|\lambda_{1}\right\rangle,\left|\lambda_{2}\right\rangle, \cdots\left|\lambda_{d}\right\rangle\right\}$ with $\left|\lambda_{d}\right\rangle$ as an absorbing state. Let $p_{i j}$ denote the transition probability from state $\left|\lambda_{i}\right\rangle$ to state $\left|\lambda_{j}\right\rangle$ in one control step; then we get a one-step state transition probability matrix $P$ with $i j$-th entry $p_{i j}$ satisfying

$$
p_{i j}= \begin{cases}\left|\left\langle\lambda_{j}\left|U^{\lambda_{i}}\right| \lambda_{i}\right\rangle\right|^{2} & \text { for } i \neq d, \\ 0 & \text { for } i=d \text { and } j \neq d, \\ 1 & \text { for } i=d \text { and } j=d .\end{cases}
$$

Here, $U^{\lambda_{i}}$ is the matrix form of the unitary evolution operator in the eigenbasis and $\sum_{j=1}^{d} p_{i j}=1$. It should be noted that the $i$-row vector of $P$ depends only on $U^{\lambda_{i}}$ and independent of $U^{\lambda_{j}}, j \neq i$. Taking the 4-dimensional system in Figure 2 as an example, the one-step state transition probability matrix is

$$
P=\left[\begin{array}{cccc}
\left|\left(U^{\lambda_{1}}\right)_{11}\right|^{2} & \left|\left(U^{\lambda_{1}}\right)_{21}\right|^{2} & \left|\left(U^{\lambda_{1}}\right)_{31}\right|^{2} & \left|\left(U^{\lambda_{1}}\right)_{41}\right|^{2} \\
\left|\left(U^{\lambda_{2}}\right)_{12}\right|^{2} & \left|\left(U^{\lambda_{2}}\right)_{22}\right|^{2} & \left|\left(U^{\lambda_{2}}\right)_{32}\right|^{2} & \left|\left(U^{\lambda_{2}}\right)_{42}\right|^{2} \\
\left|\left(U^{\lambda_{3}}\right)_{13}\right|^{2} & \left|\left(U^{\lambda_{3}}\right)_{23}\right|^{2} & \left|\left(U^{\lambda_{3}}\right)_{33}\right|^{2} & \left|\left(U^{\lambda_{3}}\right)_{43}\right|^{2} \\
0 & 0 & 0 & 1
\end{array}\right] .
$$

Here, $\quad p_{i j}=\left|\left(U^{\lambda_{i}}\right)_{j i}\right|^{2}$ is the modulus-square of the $j i$-th entry of $U^{\lambda_{i}}$. We could rewrite $P$ into block-matrix form:

$$
P=\left[\begin{array}{cc}
Q & R \\
O & 1
\end{array}\right]
$$


where $Q$ is a $(d-1) \times(d-1)$ matrix with $i j$-th entry $q_{i j}=p_{i j}, R$ is a column vector with $i$-th entry $r_{i}=p_{i d}$, and $O$ is a zero row vector.

From Markov chain theory, we can recast the $k$-step state transition probability matrix $P^{(k)}$ into block-matrix form:

$$
P^{(k)}=P^{k}=\left[\begin{array}{cc}
Q^{k} & R^{(k)} \\
O & 1
\end{array}\right],
$$

where $R^{(k)}=\left(I+Q+Q^{2}+\cdots+Q^{k-1}\right) R$. Since $\left|\lambda_{d}\right\rangle$ is an absorbing state of the Markov chain, the $i$-th entry $r_{i}^{(k)}$ of $R^{(k)}$ denotes the transition probability from state $\left|\lambda_{i}\right\rangle$ to the target eigenstate $\left|\lambda_{d}\right\rangle$ in $k$ steps. It should be noted that the probability includes attaining $\left|\lambda_{d}\right\rangle$ in less than $k$ steps.

From the view of Markov chain, the argument that a state $\left|\lambda_{j}\right\rangle$ is reachable from $\left|\lambda_{i}\right\rangle$ means that there exists $m \geqslant 1$ such that the $m$-step transition probability $p_{i j}^{(m)}>0$. Using these ideas, we have the following propositions and lemmas based on the analysis of absorbing Markov chains developed in [24].

Proposition 3.2. (Finite-step Reachability with Probability) If the target eigenstate is reachable from all eigenstates, for any given probability $p \in(0,1)$, there exists a finite $k$ such that after $k$ steps the state has reached the target eigenstate with probability greater than $p$.

Proof. If the target eigenstate is reachable from all eigenstates in the sense of Markov chain, then for $\forall i \in\{1$, $2, \ldots d-1\}$, there exist finite numbers $m_{i}$ and positive numbers $p_{i}$, such that $r_{i}^{\left(m_{i}\right)}>p_{i}>0$.

Let $m=\max _{i} m_{i}$ and $p_{m}=\min _{i} p_{i}$. Since $r_{i}^{(k+1)}=r_{i}^{(k)}+\left(Q_{k} R\right)_{i} \geqslant$ $r_{i}^{(k)}$, for all initial eigenstates, after $m$ steps, the system will reach the target eigenstate with probability greater than $p_{m}$. In other words, the probability that the system will not reach the target eigenstate after $m$ steps is less than $1-p_{m}<1$. If the system does not reach the target eigenstate after $2 m$ steps, it cannot reach the target eigenstate in $m$ steps and cannot reach the target eigenstate in the next $m$ steps. Thus the probability that it does not reach the target eigenstate after $2 m$ steps is less than $\left(1-p_{m}\right)^{2}$. Analogously, the probability that it does not reach the target eigenstate after $l \times m$ steps is less than $\left(1-p_{m}\right)^{l}$.

For $\forall p \in(0,1)$, choose $l$ large enough to make sure $\left(1-p_{m}\right)^{l}<1-p$, and let $k$ be an integral number not less than $m \times \log _{\left(1-p_{m}\right)}(1-p)$. Then, after $k$ steps, the state will reach the target eigenstate with probability greater than $p$.

The above proposition shows that the state of the controlled system will converge to the target eigenstate with probability one after finite control steps. However, the more control steps used, the higher the cost. Hence, we need to optimize the unitary evolution to reach the target with less number of control steps.

Lemma 3.3. If the target eigenstate is reachable from all eigenstates, then $I-Q$ is nonsingular and

$$
(I-Q)^{-1}=\sum_{k=0}^{\infty} Q^{k}
$$

Proof. If the target eigenstate is reachable from all eigenstates, from the proof of the above proposition, we could conclude that after $m$ steps, the probability of reaching the target eigenstate from state $\left|\lambda_{i}\right\rangle$ is $r_{i}^{(m)}>p_{m}>0$; thus the sum of the entries of any row in $Q^{m}$ is less than 1 . As a result, we have $\lim _{k \rightarrow \infty} Q^{k}=0$.

Let $(I-Q) x=0$, that is $x=Q x$. By iteration, we have $x=Q^{n} x$. Since $Q^{n} \rightarrow 0$, we have $Q^{n} x \rightarrow 0$. Hence the only solution is $x=0$. Thus $I-Q$ is nonsingular. Note that

$$
(I-Q)\left(I+Q+Q^{2}+\cdots+Q^{k}\right)=I-Q^{k+1} .
$$

Letting $k$ tend to infinity we have

$$
(I-Q)^{-1}=\sum_{k=0}^{\infty} Q^{k}
$$

Define $N=(I-Q)^{-1}$ as the fundamental matrix for $P$. From [25], we have the following lemma.

Lemma 3.4. The $i j$-th entry $n_{i j}$ of the matrix $N$ is the expected number of steps that the chain is in state $\left|\lambda_{j}\right\rangle$ before being absorbed into the target eigenstate if it starts in state $\left|\lambda_{i}\right\rangle$.

Proof. Let $X^{(k)}$ be a random variable equaling 1 if the chain is in state $\left|\lambda_{j}\right\rangle$ after $k$ steps, and 0 otherwise. We have

$$
\begin{gathered}
\operatorname{Prob}\left(X^{(k)}=1\right)=q_{i j}^{(k)}, \\
\operatorname{Prob}\left(X^{(k)}=0\right)=1-q_{i j}^{(k)},
\end{gathered}
$$

where $q_{i j}^{(k)}$ is the $i j$-th entry of $Q^{k}$. Since $X^{(k)}$ is a $0-1$ random variable, $E\left(X^{(k)}\right)=q_{i j}^{(k)}$. The expected number of steps for which the chain is in state $\left|\lambda_{j}\right\rangle$ in the first $k$ steps, given that it starts in state $\left|\lambda_{i}\right\rangle$, is clearly

$$
E\left(X^{(0)}+X^{(1)}+\cdots+X^{(k)}\right)=q_{i j}^{(0)}+q_{i j}^{(1)}+\cdots+q_{i j}^{(k)} .
$$

Letting $k$ tend to infinity we have

$$
E\left(X^{(0)}+X^{(1)}+\cdots\right)=q_{i j}^{(0)}+q_{i j}^{(1)}+\cdots=n_{i j} .
$$

The $i j$-th entry $n_{i j}$ of $N$ gives the expected number of steps that the process is in the state $\left|\lambda_{j}\right\rangle$ from initial state $\left|\lambda_{i}\right\rangle$ before being absorbed. Hence the sum of the entries of the $i$-th row of $N$ gives the expected number of steps before being absorbed into the target eigenstate from the initial state $\left|\lambda_{i}\right\rangle$.

Proposition 3.5. (Least Step-number Condition) For the initial eigenstate $\left|\lambda_{1}\right\rangle$ and target eigenstate $\left|\lambda_{d}\right\rangle$, if $U^{\lambda_{i}}$, 
$i=1,2, \ldots d-1$, is chosen to minimize the sum of entries of the first row of $N$, the controlled system can reach the target eigenstate with the least number of steps.

Proof. Since the $i j$-th entry $n_{i j}$ of $N$ gives the expected number of steps that the process is in state $\left|\lambda_{j}\right\rangle$ from initial state $\left|\lambda_{i}\right\rangle$ before being absorbed, the sum of the entries of the first row of $N$ gives the expected number of steps before being absorbed if it starts from initial state $\left|\lambda_{1}\right\rangle$. As a result, if the control action is chosen to minimize the sum of the entries of the first row of $N$, the controlled system can reach the target eigenstate with the least number of control steps in expectation.

The above proposition gives a least step-number condition to steer a system to the target eigenstate. In our scheme, the control action is chosen based on the output eigenstate of the projective measurement. The $i$-th row of $P$ depends only on the corresponding control action conditioned on the $i$-th output eigenstate of the measurement. Hence, we have $d-1$ control actions $\left\{U^{\lambda_{1}}, U^{\lambda_{2}}, \ldots U^{\lambda_{d-1}}\right\}$. The control action group forms the state transition probability matrix $P$. To reach the target with the least number of control steps, we choose the best control action group according to the least step-number condition.

\section{Analysis of simulated realization}

In Section 3, we have presented the scheme of multi-step evolution and measurement control in theory. However, for most quantum systems in practice, it is difficult to get an analytical solution. Hence, the numerical solution is discussed in this section and some experimental simulations with discussions will be presented in the next section.

\subsection{Approximation to least step-number condition}

Since the relation between the control action and the sum of the entries of the first row of $N$ is not clear enough to guide us to choose the best control action, we take an approximation to get a simpler and easier way to choose the control action.

As $N(Q)=I+Q+Q^{2}+Q^{3}+\cdots$ and the norm of matrix $Q^{k}$ decreases as $k$ grows, we could take the sum of finite sequences of $Q^{k}$ to approximate $N$. We call

$$
S(Q, k)=I+Q+Q^{2}+Q^{3}+\cdots+Q^{k}
$$

as the $k$-th order approximation of $N(Q)$. The corresponding expected steps before being absorbed is

$$
T(Q, k)=e_{1}^{T} S(Q, k) c,
$$

where $e_{1}^{T}=\left[\begin{array}{llll}1 & 0 & \cdots & 0\end{array}\right]$ and $c^{T}=\left[\begin{array}{llll}1 & 1 & \cdots & 1\end{array}\right]$.

The first-order approximation $T(Q, 1)$ is

$$
T(Q, 1)=e_{1}^{T}(I+Q) c=1+\left(1-p_{1 d}\right) .
$$

In this case, we maximize $p_{1 d}$ to minimize the expected number of steps before being absorbed. In other words, we choose the control action for which the one-step transition probability is the largest to transmit the initial eigenstate to the target eigenstate.

The second-order approximation $T(Q, 2)$ is

$$
\begin{aligned}
T(Q, 2) & =e_{1}^{T}\left(I+Q+Q^{2}\right) c=1+\sum_{j=1}^{d-1} p_{1 j}+\sum_{j=1}^{d-1} \sum_{k=1}^{d-1} p_{1 k} p_{k j} \\
& =3-2 p_{1 d}-\sum_{k=1}^{d-1} p_{1 k} p_{k d} .
\end{aligned}
$$

To minimize $T(Q, 2)$, we just need to maximize $2 p_{1 d}+\sum_{k=1}^{d-1} p_{1 k} p_{k d}$. Note that $p_{k d}, k=1,2, \ldots d-1$, are independent of each other and of other factors involved in $T(Q, 2)$. Hence we could choose the unitary evolution matrix $U^{\lambda_{k}}$ to maximize $p_{k d}$ once the output state of the measurement is $\left|\lambda_{k}\right\rangle$. However, for $U^{\lambda_{1}}$, the optimal problem becomes hard to treat. As $\sum_{k=1}^{d} p_{1 k}=1$ and the coefficient of $p_{1 d}$ is always larger than $p_{1 k}, k \neq d$, we could change $U^{\lambda_{1}}$ to maximize $p_{1 d}$ and get a fairly good solution but that might not be the best. As a result, the control law is to choose the control action for which the one-step transition probability is the largest for transition to the target eigenstate from the output eigenstate of the measurement in each control step.

\subsection{Discretization of control strengths}

To get a numerical solution, we constrain the controlled system to the following bilinear system [22]:

$$
\mathrm{i} \hbar|\dot{\psi}(t)\rangle=\left(H_{0}+\sum_{m=1}^{M} u_{m}^{\lambda_{i}}(t) H_{m}\right)|\psi(t)\rangle,
$$

where $H_{0}$ is the drift Hamiltonian and $H_{m}$ is the control Hamiltonian. $u_{m}^{\lambda_{i}}(t)$ is the control strength of $H_{m}$ if the output eigenstate of the measurement at last step is $\left|\lambda_{i}\right\rangle$. In addition, we fix the unitary evolution time $T^{\lambda_{i}}$ of each control step to $T$ and keep the control strengths $\left\{u_{m}^{\lambda_{i}}(t)\right\}$ invariant during a control step.

In these settings, after recalling the derivation process of the state transition probability, we could easily conclude its differentiability with respect to the control strengths.

Proposition 4.1. The state transition probability matrix is differential with respect to the control strengths.

Proof. The Hamiltonian of the bilinear quantum system under control conditioning on the output state of the measurement $\left|\lambda_{i}\right\rangle$ can be written as

$$
H^{\lambda_{i}}=H_{0}+\sum_{m=1}^{M} u_{m}^{\lambda_{i}} H_{m} .
$$


The unitary evolution of the state can be represented as matrix form

$$
U^{\lambda_{i}}=\exp \left(-\frac{\mathrm{i} T}{\hbar}\left(H_{0}+\sum_{m=1}^{M} u_{m}^{\lambda_{i}} H_{m}\right)\right)
$$

The projective measurement will collapse the state $U^{\lambda_{i}}\left|\lambda_{i}\right\rangle$ to one of its eigenstates $\left|\lambda_{j}\right\rangle$ with probability $\left|\left(U^{\lambda_{i}}\right)_{j i}\right|^{2}$. The one-step state transition probability from eigenstate $\left|\lambda_{i}\right\rangle$ to eigenstate $\left|\lambda_{j}\right\rangle$ will be

$$
p_{i j}=\left|\left(U^{\lambda_{i}}\right)_{j i}\right|^{2} .
$$

Since $U^{\lambda_{i}}$ is a unitary matrix, we have $\sum_{j=1}^{d}\left|\left(U^{\lambda_{i}}\right)_{j i}\right|^{2}=1$, and thus it does not need to normalize $\left|\left(U^{\lambda_{i}}\right)_{j i}\right|^{2}$ to make sure $\sum_{j=1}^{d} p_{i j}=1$.

To illustrate the above scheme, we consider the differentiability of $P$ with respect to $u_{m}^{\lambda_{i}}$. From [25], we know $U^{\lambda_{i}}$ is differential with respect to $u_{m}^{\lambda_{i}}$ :

$$
\begin{aligned}
\frac{\partial U^{\lambda_{i}}}{\partial u_{m}^{\lambda_{i}}}=\frac{\mathrm{i}}{\hbar} \int_{0}^{T} & \exp \left(-\frac{\mathrm{i}(T-t)}{\hbar}\left(H_{0}+\sum_{m=1}^{M} u_{m}^{\lambda_{i}} H_{m}\right)\right) \\
& \times H_{m} \exp \left(-\frac{\mathrm{i} t}{\hbar}\left(H_{0}+\sum_{m=1}^{M} u_{m}^{\lambda_{i}} H_{m}\right)\right) \mathrm{d} t .
\end{aligned}
$$

Although the modulus of a complex number is not differential with respect to the complex number, as the complex number $\left(U^{\lambda_{i}}\right)_{j i}$ is related to a single real parameter $u_{m}^{\lambda_{i}}$, the differentiability of $P$ with respect to $u_{m}^{\lambda_{i}}$ holds as shown below. We could rewrite $\left(U^{\lambda_{i}}\right)_{j i}=f_{j i}\left(u_{m}^{\lambda_{i}}\right)+$ $\mathrm{i} g_{j i}\left(u_{m}^{\lambda_{i}}\right)$, where $f_{j i}, g_{j i}$ are both real functions and are differential with respect to $u_{m}^{\lambda_{i}}$.

$$
\frac{\partial p_{i j}}{\partial u_{m}^{\lambda_{i}}}=2 f_{j i}\left(u_{m}^{\lambda_{i}}\right) \frac{\mathrm{d} f_{j i}\left(u_{m}^{\lambda_{i}}\right)}{\mathrm{d} u_{m}^{\lambda_{i}}}+2 g_{j i}\left(u_{m}^{\lambda_{i}}\right) \frac{\mathrm{d} g_{j i}\left(u_{m}^{\lambda_{i}}\right)}{\mathrm{d} u_{m}^{\lambda_{i}}} .
$$

Hence the state transition probability matrix is differential with respect to the control strengths.

Based on the above proposition, we can deduce the probability matrix is uniformly continuous with respect to the control strengths. Thus, we can discretize the control strengths with errors under tight control. For a better numerical solution, we could discretize the control strengths more densely in the expense of a larger computation. However we need not be concerned that there might exist singular points with very large errors.

To apply our scheme in practice, we first discretize the control strengths which are generally bounded and get a series of control strength groups $\left\{\left(u_{1}^{k_{1}}, u_{2}^{k_{2}}, \cdots u_{M}^{k_{M}}\right)\right\}$. Then, we calculate the state transition probability matrix within these control strength groups and search for the best group $\left(u_{1}^{\lambda_{i}}, u_{2}^{\lambda_{i}}, \cdots u_{M}^{\lambda_{i}}\right)$ to transmit the system from the output eigenstate $\left|\lambda_{i}\right\rangle$ to the target eigenstate with the least number of control steps. To simplify the operation, we could make a first- or second-order approximation for a calculation of the expected number of steps. In operation, we first make the projective measurement on the system. If the output eigenstate is not the target eigenstate, we choose an appropriate control strength group for the output eigenstate of the measurement that when applied, enables controlled evolution of the system. For example, if the output eigenstate is $\left|\lambda_{i}\right\rangle$, we choose the control strength group $\left(u_{1}^{\lambda_{i}}, u_{2}^{\lambda_{i}}, \cdots u_{M}^{\lambda_{i}}\right)$. After evolution for a period of time $T$, we make a projective measurement on the system again. If the output state is just the target eigenstate, we finish the control process successfully; otherwise, we continue the above control process until the target eigenstate is achieved.

\section{Simulated experiments and discussions}

We now consider two coupled qubits formed from the spins of a nuclear and an electron. The drift Hamiltonian is

$$
H_{0}=S_{z}+0.5 I_{z}+0.1 S_{z} I_{z},
$$

where $S_{z}=\frac{1}{2} \sigma_{z} \otimes I_{2}$ and $I_{z}=\frac{1}{2} I_{2} \otimes \sigma_{z}$. We choose the control Hamiltonians to be $H_{1}=S_{x}=\frac{1}{2} \sigma_{x} \otimes I_{2} \quad$ and $H_{2}=I_{x}=\frac{1}{2} I_{2} \otimes \sigma_{x}$. Here $\sigma_{x}=\left[\begin{array}{ll}0 & 1 \\ 1 & 0\end{array}\right], \quad \sigma_{z}=\left[\begin{array}{cc}1 & 0 \\ 0 & -1\end{array}\right]$, $I_{2}=\left[\begin{array}{ll}1 & 0 \\ 0 & 1\end{array}\right]$ are the Pauli matrices. The evolution of this quantum system can be formulated as

$$
i|\dot{\psi}(t)\rangle=\left(H_{0}+u_{1} H_{1}+u_{2} H_{2}\right)|\psi(t)\rangle,
$$

where we assume $\hbar=1$ for simplicity. Under constraint conditions described in Section 4.2, we make the following simulation experiments.

If we leave the Hamiltonian unchanged during each step of evolution, we discretize the control strengths and simulate the control process on 10000 samples. In this way, we obtain the number of steps required to transform the initial state to the target eigenstate for all samples. We then can calculate the number of steps required with probability greater than 0.98. This is plotted with respect to the control strengths in Figure 3. From the numerical solution we can find an appropriate control action so that the system evolves with the least number of steps.

If we perform a second-order approximation to obtain the 
multi-step evolution and measurement control to be applied on the system with $u_{m}^{\lambda_{i}} \in[-5,5]$, we can calculate the control law quickly and simulate the control process with a Monte Carlo method on 10000 samples. The result is shown in Figure 4 where most of the samples finish on the target eigenstate within two steps. Hence, a second-order approximation can perform the process within fewer steps.

We have also compared the expected number of steps required from first- and second-order approximations. We choose here $u^{\lambda_{i}} \in[-2,2]$, and again simulate the control process on 10000 samples. We plot sample counts reaching the target eigenstate with respect to the number of control steps in Figure 5. The system finishes up in the target eigenstate in less number of steps in the second-order approximation than for first-order approximation.

From the experimental results, one can see the validity of our scheme to steer the system to a target eigenstate. If the free degree of the control action is large, the number of control steps to reach the target eigenstate with a certain

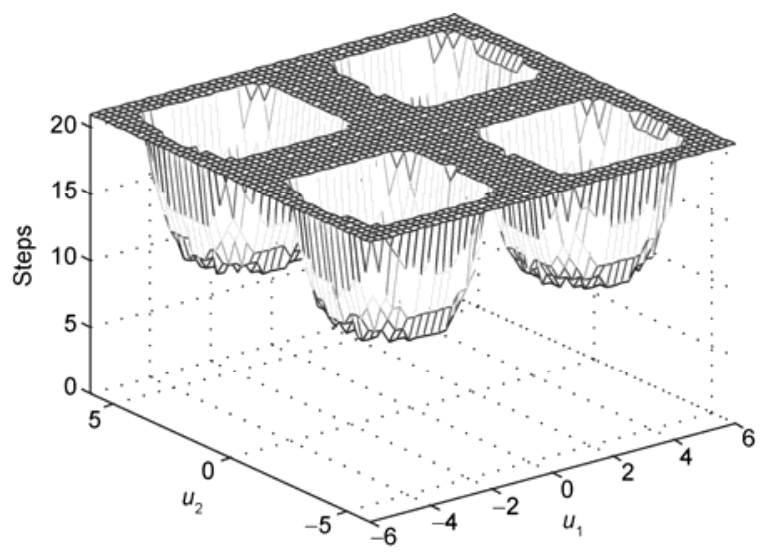

Figure 3 Steps required to steer the system from initial state $\left|\lambda_{1}\right\rangle$ to target eigenstate $\left|\lambda_{4}\right\rangle$ with respect to control strengths $\left(u_{1}, u_{2}\right)$ if the control strengths are kept constant during each step (the maximum number of steps in the simulation is set to 20 ).

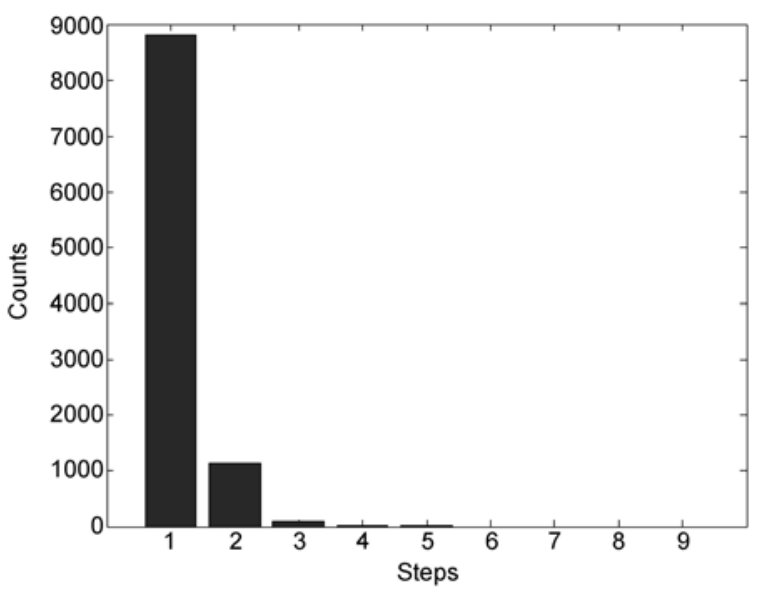

Figure 4 Sample counts reaching the target eigenstate with respect to the control steps in the second-order approximation.
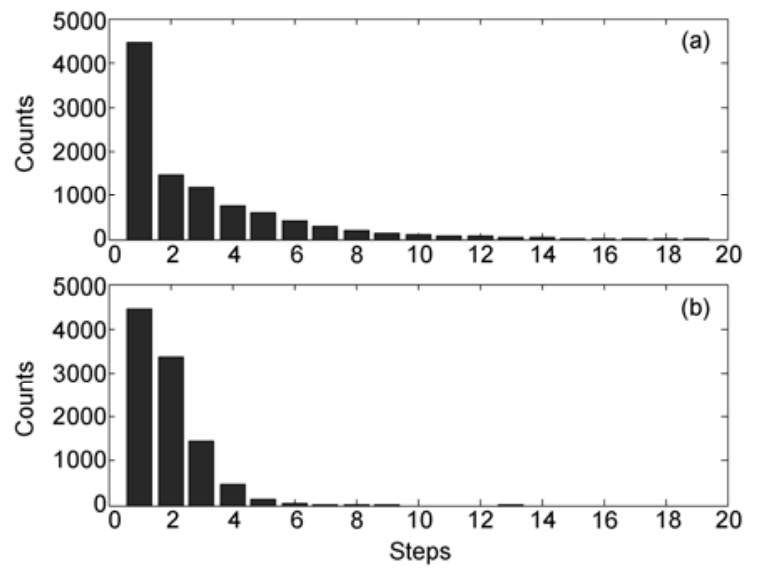

Figure 5 Sample counts reaching the target eigenstate with respect to the control steps in the first-order (a) and second-order (b) approximation (the maximum number of steps in the simulation is set to 18 ).

probability could be very small after using the optimized algorithm. Although multi-control steps are required in our scheme, we just need to measure one observable many times. This differs from the scheme proposed by Pechen et al. [13] which needs to measure a set of different observables one by one. The control law of our scheme is simple and is easy to realize for quantum spin systems using Stern-Gerlach measurements or for photons in quantum optical systems with beam splitters as measurement devices. For a $d$-dimensional quantum system, we need only calculate $d-1$ unitary evolution operators off-line to apply during system evolution. With first-order approximations, only one unitary evolution operator is required; with second-order approximation, the unitary evolution operator is chosen based on the output eigenstate of the measurement. As the control steps go on, the probability to reach the target eigenstate will increase; this is an aspect which is different from the scheme in [11]. In that scheme, we only perform the measurement once, but the algorithm then has a probability of failure. Here the target eigenstate can be always reached after a finite number of control steps. The cost is that we need to repeat the control step many times until the target is achieved.

\section{Conclusion}

In this paper, we have proposed a simple and easy quantum control scheme to steer finite-dimensional quantum systems from any initial state to one of its eigenstates by performing in turn unitary evolution and projective measurement. We have investigated the control process using Markov chains, proven its finite-step reachability with unit probability, and created an algorithm giving the least number of steps to reach the target eigenstate. The main advantage of this scheme is that it is designed off-line and can be easily applied to practical systems. This scheme has potential appli- 
cations to state preparation [26], decoherence-free subspaces [27] and sliding mode control [28]. Since measurement could weaken conditions required for unitary evolution control [9], the scheme can also serve as an intermediate process to steer a quantum system to any pure state in its corresponding Hilbert space.

This work was supported by the National Natural Science Foundation of China (60804020).

1 Brif C, Chakrabarti R, Rabitz H. Control of quantum phenomena: Past, present, and future. New J Phys, 2010, 12: 075008

2 Dong D, Petersen I R. Quantum control theory and applications: A survey. IET Control Theory Appl, 2010, 4: 2651-2671

3 Huang G M, Tarn T J. On the controllability of quantum mechanical systems. J Math Phys, 1983, 24: 2608

4 Werschnic J, Gross E K U. Quantum optimal control theory. J Phys B At Mol Opt Phys, 2007, 40: R175

5 Peirce A P, Dahleh M A. Optimal control of quantum mechanical systems: Existence, numerical approximation, and applications. Phys Rev A, 1988, 37: 4950-4964

6 Rabitz H, de Vivie-Riedle R, Motzkus M, et al. Whither the future of controlling quantum phenomena? Science, 2000, 288: 824-828

7 Judson R S, Rabitz H. Teaching lasers to control molecules. Phys Rev Lett, 1992, 68: 1500-1503

8 Doherty A C, Jacobs K. Feedback control of quantum systems using continuous state estimation. Phys Rev A, 1999, 60: 2700-2711

9 Mendes R V, Man'ko V I. Quantum control and the Strocchi map. Phys Rev A, 2003, 67: 053404

10 Mandilara A, Clark J W. Probabilistic quantum control via indirect measurement. Phys Rev A, 2005, 71: 013406

11 Zhang C B, Dong D, Chen Z H. Control of non-controllable quantum systems: A quantum control algorithm based on Grover iteration. J Opt B: Quant Semiclass Opt, 2005, 7: S313-S317

12 Roa L, Delgada A, Guevara M L L, et al. Measurement driven quantum evolution. Phys Rev A, 2006, 73: 012322
13 Pechen A, Il'in N, Shuang F, et al. Quantum control by von Neumann measurements. Phys Rev A, 2006, 74: 052102

14 Dong D, Chen C L, Tarn T J, et al. Incoherent control of quantum systems with wavefunction controllable subspaces via quantum reinforcement learning. IEEE Trans Syst Man Cybern B: Cybern, 2008, 38: 957-962

15 Dong D, Zhang C B, Rabitz H, et al. Incoherent control of locally controllable quantum systems. J Chem Phys, 2008, 129: 154103

16 Broers B, van Linder van den Heuvell H B, Noordam L D. Efficient population transfer in a three-level ladder system by frequency-swept ultrashort laser pulses. Phys Rev Lett, 1992, 69: 2062-2065

17 Bergmann K, Theuer H, Shore B W. Coherent population transfer among quantum states of atoms and molecules. Phys Rev Mod, 1998, 70: $1003-1025$

18 Zhdanovich S, Shapiro E A, Hepburn J W, et al. Population transfer between two quantum states by piecewise chirping of femtosecond pulses: theory and experiment. Phys Rev Lett, 2008, 100: 103005

19 Cao W C, Liu X S, Bai H B, et al. Bang-bang control suppression of amplitude damping in a three level atom. Sci China Ser G: Phys Mech Astron, 2008, 51: 29-37

20 Dong D, Lam J, Petersen I R. Robust incoherent control of qubit systems via switching and optimisation. Int J Control, 2010, 83: 206-217

21 Rong X, Wang Y, Yang J H, et al. Dynamical decoupling of electron spins in phosphorus-doped silicon. Chin Sci Bull, 2011, 56: 591-597

22 D'Alessandro D. Introduction to Quantum Control and Dynamics. Boca Raton: Chapman and Hall, 2008

23 Wiseman H M, Milburn G J. Quantum Measurement and Control. New York: Cambridge University Press, 2009

24 Grinstead C M, Snell J L. Introduction to probability. Providence: American Mathematical Society Press, 1997

25 Wilcox R M. Exponential operators and parameter differentiation in quantum physics. J Math Phys, 1967, 8: 962-982

26 Handel R, Stockton J K, Mabuchi H. Modelling and feedback control design for quantum state preparation. J Opt B: Quant Semiclass Opt, 2005, 7: S179

27 Wu L A, Lidar D A. Creating decoherence-free subspaces with strong and fast pulses. Phys Rev Lett, 2002, 88: 207902

28 Dong D, Petersen I R. Sliding mode control of quantum systems. New J Phys, 2009, 11: 105033

Open Access This article is distributed under the terms of the Creative Commons Attribution License which permits any use, distribution, and reproduction in any medium, provided the original author(s) and source are credited. 